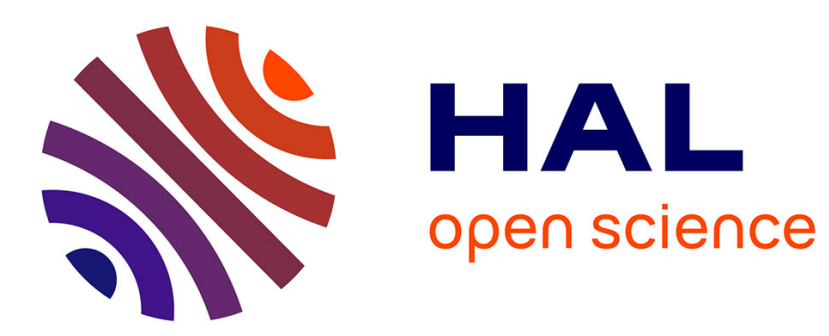

\title{
Understanding the emergence of drawing behaviour with age: a multi-metric analysis
}

\author{
Lison Martinet, Cédric Sueur, Benjamin Beltzung, Marie Pelé
}

\section{To cite this version:}

Lison Martinet, Cédric Sueur, Benjamin Beltzung, Marie Pelé. Understanding the emergence of drawing behaviour with age: a multi-metric analysis. 2021. hal-03334153

\section{HAL Id: hal-03334153 \\ https://hal.science/hal-03334153}

Preprint submitted on 3 Sep 2021

HAL is a multi-disciplinary open access archive for the deposit and dissemination of scientific research documents, whether they are published or not. The documents may come from teaching and research institutions in France or abroad, or from public or private research centers.
L'archive ouverte pluridisciplinaire HAL, est destinée au dépôt et à la diffusion de documents scientifiques de niveau recherche, publiés ou non, émanant des établissements d'enseignement et de recherche français ou étrangers, des laboratoires publics ou privés. 
Understanding the emergence of drawing behaviour with age: a multi-metric analysis

Lison Martinet ${ }^{1, *}$, Cédric Sueur $^{1,2, *},{ }^{*}$ Benjamin Beltzung ${ }^{1}$, Marie Pelé $^{3}$

1: Université de Strasbourg, CNRS, IPHC UMR 7178, Strasbourg, France

2 : Institut Universitaire de France, Paris, France

3 : Anthropo-Lab, ETHICS EA7446, Lille Catholic University, Lille, France

* Authors contributed equally

Corresponding author: Cédric Sueur, cedric.sueur@iphc.cnrs.fr; IPHC UMR 7178, 23 rue

Becquerel 67087 Strasbourg, France

\begin{abstract}
We need specific and objective methods to analyse the temporal changes of drawing in children, especially those too young to communicate via verbalisations. We asked 134 children, ranging from three to ten years old, and 38 adults to draw on a tablet under two conditions: free drawing and selfportrait. We then used seven metrics from three categories (spatial, temporal, and colorimetric) in a principal component analysis (PCA). Three dimensions of the PCA explained $77 \%$ of the variance in the drawings. We named these dimensions as diversity, sequentiality, and efficiency, which provided a mechanism for better understanding the intentionality and representativeness behind drawing. Gender had no effect, but age influenced all three dimensions differently. This multi-metric approach is a powerful tool for investigating the ontogenetic development of drawing, and could be used to understand the evolution of this behaviour by applying it to the study of primates, or to reveal drawing characteristics in people with autism and depression or those from different cultures.
\end{abstract}

Keywords: drawing behaviour; ontogenetic development; intentionality; representativeness; PCA 


\section{Introduction}

Children's drawings often attract our attention; however, these drawings may also perplex us because we do not possess the capacity to analyse their contents and understand how they are produced. The famous artist, Picasso, once said, "It took me a lifetime to paint like a child." We cannot know at what point drawings begin to have some meaning for a child, unless the child can explain this through verbalisation; thus, without language, we do not know if there are any aesthetic properties beyond the pictures. To analyse and understand the evolution of drawing in children, we need new specific and objective methods of analysis, such as those proposed in this paper ${ }^{1}$.

Complex mathematical metrics such as fractals are increasingly used to understand behavioural complexity and its development, regardless of the studied behaviour (e.g. grooming, diving, moving $)^{2-4}$. In previous studies, we used spatial and temporal fractals to better identify the ontogeny of drawing behaviours. By using spatial fractal analysis, we were able to show that drawing efficiency increases with age in children, before decreasing in adults who add many details ${ }^{5}$. This study also showed that humans draw more efficiently than chimpanzees, even if the chimpanzees' drawings were not random. Using temporal fractal analysis, we showed that the youngest children expressed a more stereotypical drawing behaviour than adults in their alternation between drawing and interruption phases; therefore, their behaviour is less complex ${ }^{6}$. These indices were completed with the addition of other spatial (e.g. total length of lines drawn), temporal (e.g. drawing speed) and colorimetric (e.g. number of colours) metrics. Using a principal component analysis (PCA) to analyse trends across several datasets, we found that drawings can be characterised in three dimensions: efficiency, diversity and sequentiality ${ }^{7}$. First, spatial efficiency refers to drawings with few details that have a wide representation, as with emoticons ${ }^{8,9}$ or sketches ${ }^{10,11}$. Second, diversity in colour metrics assists with the visual perception of objects and materials in our environment ${ }^{12,13}$. Third, the sequence of drawing behaviours and their temporal complexity are represented by the Hurst index (i.e. a fractal temporal index). When someone draws something representative, the number of sequences increases. Furthermore, their drawing behaviour is more complex, less predictable, and the stochasticity of their alternation between drawing and non-drawing sequences also increases. These analyses differentiate children's scribbles from those of adults, a task which is impossible using the naked eye, and reveal their differences in external and internal representativeness ${ }^{5}$. Moreover, these analyses and results could represent important advances in comparative psychology and evolutionary anthropology ${ }^{1}$.

In this study, we followed the same methodology to understand how efficiency, diversity, and temporal sequentiality provide a better understanding of the emergence of intentions and representativeness in drawings with age. We asked 134 children, ranging in age from three to ten years old; and 38 adults, ranging from novices to experts in drawing, to draw a self-portrait and a free drawing on tablets with their fingers (Figure 1). We then evaluated how mathematical analyses could reveal their drawing capacities. We expect children's drawings to become more complex with increased efficiency, diversity and sequentiality as their age increases. We expect adults' drawings to have higher diversity and sequentiality, but not efficiency, as they are known to add unnecessary details that decrease drawing efficiency. 


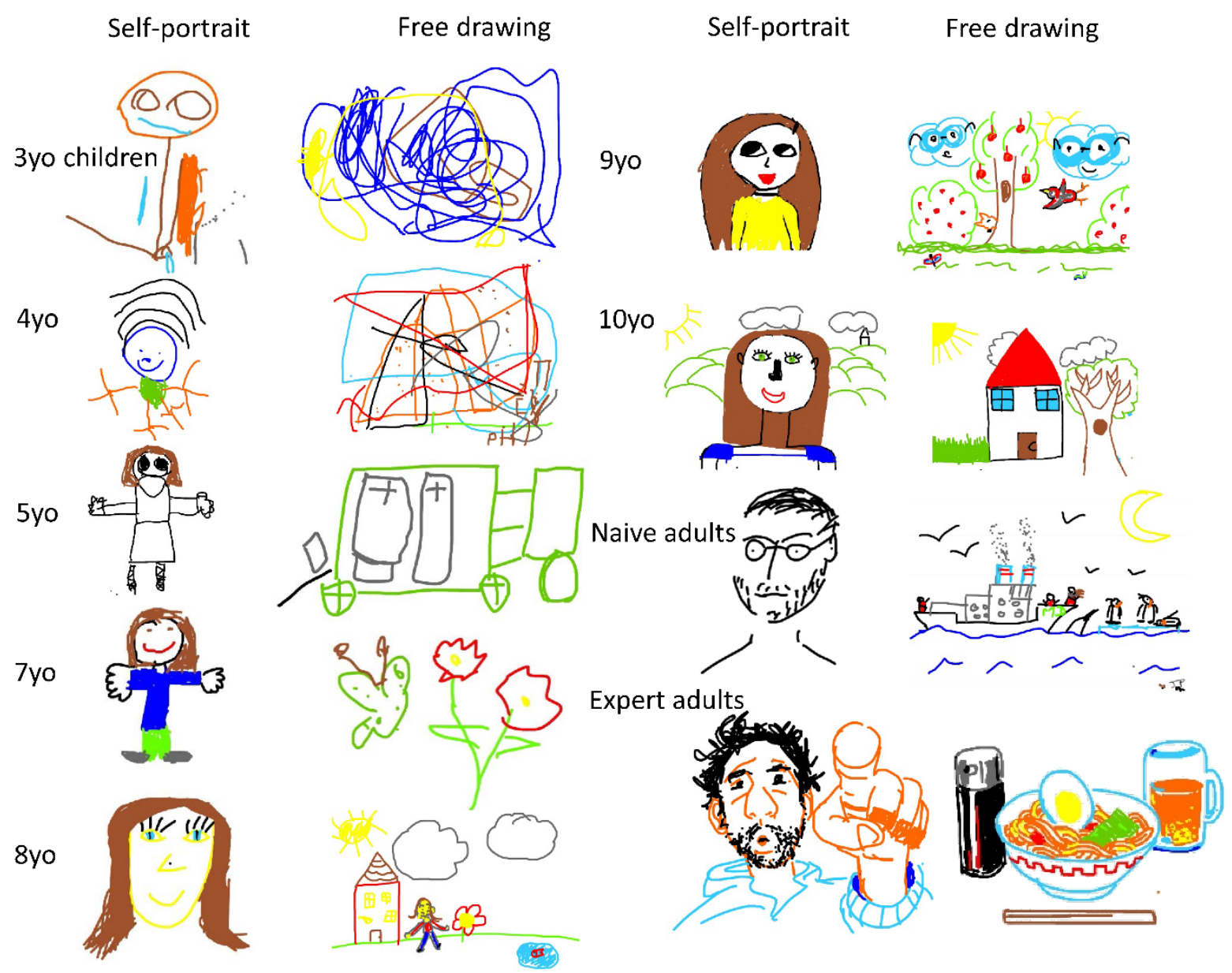

Figure 1: Representative examples of drawings created by children and adults of each age-category under free drawing and self-portrait conditions.

\section{Methods}

\subsection{Dataset}

We asked 134 children and 38 adults to draw under two conditions: free drawing conditions, in which the researcher explained to the subject that they could draw whatever they wanted and they were given no further instructions; and self-portrait conditions, in which the researcher instructed the subject to draw themselves. In total, this dataset comprised 344 drawings. The 134 children ranged in age from three to ten years old. There were 18-20 children per one-year age group with even numbers of boys and girls, except for the youngest age group which had 5 girls and 15 boys. The 38 adults comprised 19 men and 19 women, ranging in age from 21 to 60 years old, representing both novices and experts. In contrast to novices, experts were enrolled in art schools or were professional illustrators by trade. This dataset was collected during 2018 and 2019. Drawings from kindergarten children (3-5 years of age) and primary school children were collected in 2018 and 2019 , respectively. Children that were 6 years old in 2019 could not be tested because they had already been involved as 5 -year-olds in 2018. For detailed methods and more information, please refer to Martinet et al. (2021) .

\subsection{Experimental design}


The habituation phase is detailed in the Supplementary Material. During the testing phase, each child was individually tested at their school during normal class hours. Tests were conducted in their classroom for 3-year-olds, and in the staff room for older children. The researcher stayed during the test but kept their distance during drawing to avoid influencing the child. Novice adults were tested individually in a room at the research institute; experts were tested at the relevant art school. Adult participants were left alone in the room. A camera recorded their hand movements while drawing, in case we needed to control for any issues during the session; such as drawing interruption or involuntary tracing. No time limit was applied.

\subsection{Data analysis}

The software developed for these studies allowed the recording of spatial coordinates $(X ; Y)$ for every point of the lines drawn; their time coordinates (min; s; ms); and the colour for each drawing. From this data, we calculated the spatial, temporal and colorimetric metrics for each drawing (Table 1). Details about these metrics, their calculation, and the range of expected values for each are given in Sueur et al. (2021) ${ }^{7}$.

\subsection{Statistical analysis}

We followed the analyses described in Sueur et al. (2021) 7 . This methodology used a PCA to determine 14 metrics which can be used to understand drawing complexity; and was validated using two drawing datasets. This allowed the selection of the seven metrics used in this study. Most of the variables were influenced by the drawing test duration metric, which could lead to bias in the analyses. To solve this problem, we corrected all of them by performing a linear regression with each metric as the response variable and the drawing test time as a factor. We collated the residuals from this linear regression, which correspond to the variance of each point that is not explained by the drawing test duration. Then, we performed a PCA (for more details, see Supplementary Material). From the PCA results, we extracted the value of each dimension for each individual and built a new dataset.

After normalising this data, we ran Generalised Linear Models (GLMs) with each dimension as the dependent variable and the gender of participants, age categories, and conditions as independent variables. We also added the interactions of gender-condition and group-condition as independent variables to the model. Normality and homoscedasticity of residuals were verified graphically. We then performed pairwise comparisons with Benjamini-Hochberg ${ }^{14}$ correction for significant independent variables. All analyses were performed in RStudio 1.4.1103 ${ }^{15,16}$. The significance threshold was set to $\alpha=0.05$. 
Table 1: Definitions and contextual explanations of metrics used to understand drawing complexity.

\begin{tabular}{|c|c|c|c|c|}
\hline Type & Metric & Definition & Explanation & References \\
\hline \multirow{2}{*}{ 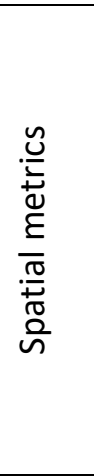 } & $\mu \mathrm{MLE}$ & $\begin{array}{l}\text { Spatial fractal metric; maximum } \\
\text { estimate power coefficient of the } \\
\text { drawing length's distribution }\end{array}$ & $\begin{array}{l}\text { It measures the drawing efficiency, from } \\
\text { random trajectories to optimal ones, and } \\
\text { indicates a representativeness (internal or } \\
\text { external) }\end{array}$ & $3,29-31$ \\
\hline & Drawing distance & $\begin{array}{l}\text { Total distance of drawing from the } \\
\text { first point to the last point; measured } \\
\text { in pixels }\end{array}$ & $\begin{array}{l}\text { We expect long-distance drawings to be more } \\
\text { representative or have more details compared } \\
\text { to short-distance drawings. However, this } \\
\text { metric can also represent deterministic drawing } \\
\text { (i.e., no intention by the subject to represent } \\
\text { anything) }\end{array}$ & $32-34$ \\
\hline \multirow{3}{*}{ 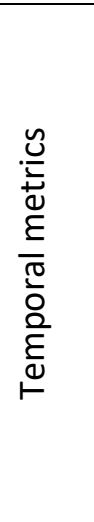 } & Hurst index & $\begin{array}{l}\text { Temporal fractal metric; measure of } \\
\text { the long-term process in the temporal } \\
\text { sequence }\end{array}$ & $\begin{array}{l}\text { It measures the temporal complexity of } \\
\text { drawings sequences, ranging from deterministic } \\
\text { to complex }\end{array}$ & 2,35 \\
\hline & Number of sequences & $\begin{array}{l}\text { Number of drawing and non-drawing } \\
\text { sequences during the test }\end{array}$ & $\begin{array}{l}\text { We expect a high number of sequences to give } \\
\text { an indication regarding goal-oriented } \\
\text { behaviours; for example, intention and } \\
\text { representativeness }\end{array}$ & \\
\hline & Drawing speed & $\begin{array}{l}\text { Speed of drawing, which is the } \\
\text { drawing distance (in pixels) divided by } \\
\text { the time to complete the line or } \\
\text { drawing }\end{array}$ & $\begin{array}{l}\text { Speed is used as a measure of goal-directedness } \\
\text { or knowledge; or in this context, mastering }\end{array}$ & $3,36,37$ \\
\hline \multirow{2}{*}{ 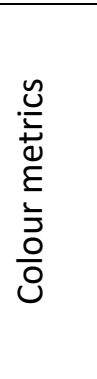 } & Mean colorimetric profile & $\begin{array}{l}\text { Mean distribution of intensity levels } \\
\text { for the red, green, or blue channels, } \\
\text { respectively; as well as removal of the } \\
\text { white (screen) colour on the parts } \\
\text { covered by the drawing }\end{array}$ & $\begin{array}{l}\text { It measures the mean spectrum of colours used, } \\
\text { from dark to light }\end{array}$ & \\
\hline & Number of colours & $\begin{array}{l}\text { Number of colours used from the ten } \\
\text { proposed colours }\end{array}$ & $\begin{array}{l}\text { We expect the number of used colours to give } \\
\text { an indication regarding intentionality, as well as } \\
\text { the interest in playing versus focused drawing }\end{array}$ & 5 \\
\hline
\end{tabular}




\section{Results}

The seven drawing metrics described in Table 1 were included in the PCA which explains $77 \%$ of the total variance (Table 2). Dimension 1, which explains $31 \%$ of the variance, contains the $\mu \mathrm{MLE}$, drawing distance and drawing speed, and corresponds to drawing efficiency. Dimension 2 (26.1\%) corresponds to the sequentiality and contains the number of sequences and the Hurst index. Dimension 3 (19.9\%) corresponds to diversity and contains the mean colorimetric profile and the number of colours used. Examples of drawings for each dimension are shown in Figures S1-S3.

Table 2: Loadings of the metrics on the three Varimax rotation PCA dimensions

\begin{tabular}{|l|r|r|r|}
\hline Dimensions & Efficiency & Sequentiality & Diversity \\
\hline$\mu \mathrm{MLE}$ & $\mathbf{0 . 8 1 4}$ & -0.246 & -0.117 \\
\hline Drawing distance & $\mathbf{- 0 . 7 4 6}$ & -0.296 & -0.371 \\
\hline Drawing speed & $\mathbf{- 0 . 9}$ & 0.216 & \\
\hline Hurst metric & 0.143 & $\mathbf{- 0 . 9 0 2}$ & \\
\hline Number of sequences & & $\mathbf{0 . 8 9 8}$ & \\
\hline Mean colorimetric profile & 0.291 & & $\mathbf{0 . 7 7 7}$ \\
\hline Number of colours & -0.178 & & $\mathbf{0 . 7 9 5}$ \\
\hline
\end{tabular}

With respect to the GLMs, the model selection of each dimension and the results for each variable are detailed in the Supplementary Material (Tables S1-S6). Interactions were only present in the models which best explained diversity, but were not significant (conditions:gender, $p=0.139$ ). Gender did not influence any of the three dimensions ( $p>0.097)$. The drawing condition only influenced the diversity dimension $(p=0.016)$, with higher values for the free drawing indicating a higher number and spectrum of colours were used. Age categories influenced all three of the dimensions (Figure 2). Pairwise comparisons (Table S7) showed that the efficiency of 3yo children was lower than that of $5 y o, 7 y o$ and 9yo children $(p<0.044)$, as was the efficiency of expert adults $(p<0.03)$. This means that we observed a decrease and then an increase with maximum efficiency around 9yo. Sequentiality (Table S8) of novice adults was higher than that of all other age categories $(p<0.004)$. Expert adults showed higher sequentiality compared to 3 yo, 4 yo and 7 yo children $(p<0.038)$. Finally, diversity (Table S9) was higher in 3yo children than in other categories $(p<0.03)$, except that of 4 yo children and novice adults. A higher diversity was noted in 4 yo children compared to that of 7yo, 9yo, 10yo children, and expert adults $(\mathrm{p}<0.004)$. A lower diversity was noted in 9yo children compared to that of 3 yo, 4yo, 5yo, 8 yo children, and novice adults $(p<0.03)$. Novice adults showed a higher diversity than 9yo children and expert adults $(p<0.03)$. There was a general decrease and then increase in the diversity dimension as young children, late-age children, and novice adults added more colours than middle-aged children and expert adults. 

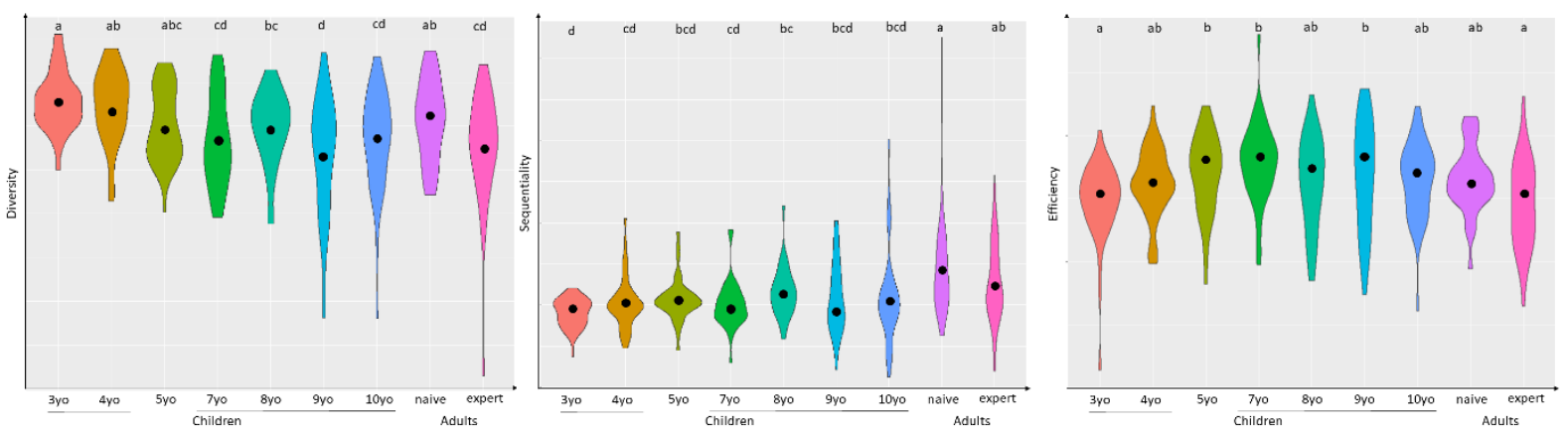

Figure 2: Violin plots representing diversity, sequentiality and efficiency across age categories. Violin plots represent the distribution of data, and the black dots represent the mean. Age groups with the same letter belong to the same group according to pairwise comparison tests (see details in Supplementary Material).

\section{Discussion}

While the final product may be non-figurative to human eyes, the process of drawing can reveal the artist's intentionality. Using a combination of different metrics, it is possible to differentiate a child's scribbles from those of an adult because even if the final product is similar, its manufacturing process is not ${ }^{7}$. In this study, we showed that the drawing dimensions identified in previous datasets - efficiency, sequentiality, and diversity - change with age.

We also tested two other factors: the gender of the participants and the conditions of the drawing sessions (i.e. free drawing or self-portrait). Gender had no significant overall effects on the drawing dimensions, barring a minor influence on sequentiality. In a previous study, Martinet et al. ${ }^{5}$ found that gender had an effect on colour use but not on the $\mu$ MLE spatial fractal metric. Other studies have shown that girls use more colours than boys in their drawings ${ }^{17,18}$, but this mainly depends on the age of the children ${ }^{19}$ and the instructions that they receive. Our results indicate that a higher number and more diverse spectrum of colours were used in free drawing versus self-portrait conditions, which may mitigate the gender effect. The open nature of free productions increased the use of colours, whereas the limitations imposed by self-portrait conditions lead individuals to use fewer elements when composing their drawing. Martinet et al. ${ }^{5}$ also found that all age groups spent more time drawing under the free condition than under the self-portrait condition. This is an important bias to consider when thinking about research protocols, as the instructions given to participants appear to constrain their drawing process and may influence the results.

As expected, age influenced the three dimensions of drawing. The first dimension, efficiency, represents the $\mu \mathrm{MLE}$ spatial fractal index, drawing distance, and drawing speed. It provides an insight into the representativeness and intentionality behind a drawing, even an abstract one; as well as the details needed to make a drawing figurative, such as the drawing distance. As such, the relationship between age and efficiency is nonlinear and efficiency initially increases, then decreases with age. This trend has previously been found with the $\mu$ MLE spatial fractal metric ${ }^{5}$, but the efficiency dimension from the PCA in this study gives more discriminative results. The efficiency increase in young children can be easily explained by the progressive development of more controlled and goaloriented lines which often underlie the production of figurative drawings. Conversely, 3yo children are more motivated by motor pleasure alone when producing scribbles ${ }^{20}$; therefore, efficiency is lowest for this age category. Thereafter, efficiency increases in 7-8yo children and decreases in 10yo children and adults. At 7-8yo, children draw all the parts of the object they have in mind without 
abstraction or unnecessary details ${ }^{20,21}$. Their primary goal is to be understood (external representativeness) with no aesthetic goals, which ensures that their drawings are more efficient. Adults' drawings appear more complex because of the compilation of numerous details.

Furthermore, adult representations may be subject to other influences, such as social norms, which young children are not ${ }^{22}$; and our results in the diversity and sequentiality dimensions confirm this. The diversity dimension represents the diversity and number of colours used. Young children are more motivated by the desire to play rather than drawing; thus, they try more colours and return the highest diversity values. Diversity is also higher among novice adults due to their willingness to add more details. Children of 7-9yo have the lowest diversity as their drawing efficiency is high. Sequentiality represents the number of sequences and the temporal complexity of alternations; and it generally increased from the youngest children to the adults. Very young children draw with scribbles using few sequences, and are more deterministic than their older counterparts. Adults tend to add many details; as such, they present the highest number of sequences and alternations between drawing and interruption resulting in a higher complexity. Therefore, the higher sequentiality in adults results in a lower efficiency. Furthermore, the study of drawing's temporal components completes the spatial analysis and allows us to further understand the ontogenetic development of drawing behaviour.

Drawing behaviour is complex, and it is impossible to understand its development through a single metric. Decades of studies are providing a combination of metrics from which we are progressively gaining a better understanding of the ontogenetic development of this behaviour. In this study, three dimensions which represent a combination of metrics allowed the objective analysis of drawings, both figurative and non-figurative ${ }^{23}$. By applying this method in studies on other primates, it could be used to better understand the evolution of this behaviour. Furthermore, this method could potentially be used to reveal the drawing characteristics and behaviour of people with autism ${ }^{24,25}$; people suffering from depression ${ }^{26,27}$; and people from different cultures ${ }^{9,28}$.

\section{Ethics statement}

We followed the ethical guidelines of our respective research institutions, and ethical approval was obtained from the Strasbourg University Research Ethics Committee (Unistra/CER/2019-11). The drawings were collected anonymously. The contributions of all participants were voluntary, and in the case of children, were subject to parental consent.

\section{Acknowledgments}

We thank the director and the teachers of the school for giving us access to their classrooms and showing interest in our research project. We are grateful to all the participants and to the parents of all the children, who accepted with enthusiasm to contribute to our study. Thanks also to Sarah Piquette, who provided help for the ethical components of this project.

\section{Data accessibility}

The data are available at the Zenodo repository (https://doi.org/10.5281/zenodo.5415290).

\section{Funding sources}

This project has received financial support from the CNRS through the MITI interdisciplinary programs and from the University of Strasbourg through an Idex Exploratory Research program.

\section{Competing interests}

We declare we have no competing interests 
Supplementary material for Understanding the emergence of drawing behaviour with age: a multimetric analysis

Lison Martinet ${ }^{1, *}$, Cédric Sueur $^{1,2, *}$, Benjamin Beltzung ${ }^{1}$, Marie Pelé $^{3}$

1: Université de Strasbourg, CNRS, IPHC UMR 7178, Strasbourg, France

2 : Institut Universitaire de France, Paris, France

3 : Anthropo-Lab, ETHICS EA7446, Lille Catholic University, Lille, France

* Authors contributed equally

Corresponding author: Cédric Sueur, cedric.sueur@iphc.cnrs.fr; IPHC UMR 7178, 23 rue

Becquerel 67087 Strasbourg, France

Habituation phase: each participant was invited to try a touchscreen tablet (iPad Pro, 13-Inch, version 11.2.2, capacitive screen reacting to the conductive touch of human fingers), then draw on it with their fingers to understand how it worked, notably to change the colour used. The drawing with fingers was preferred to involve youngest children who have not yet mastered the use of a pencil. A panel consisting of ten different colours was displayed on the bottom of the screen, and the participant could select one colour for their drawing by clicking on one of them. When they clicked on a different colour in the panel, any subsequent drawing production was in that colour. Children were habituated the day before the tests to avoid overstimulation. Adults were tested immediately after discovering the tablet.

Multi-model inferences protocol: We ran multi-model inferences with protocols to compare and rank candidate models according to (i) their respective Akaike information criterion after correction for small sample sizes (AICc) and (ii) normalized Akaike weights (AICW) ${ }^{1}$. $\triangle \mathrm{AICc}$ is the difference in AICC between one given model and the model with the lowest AIC. The AIC weight indicates the probability of a given model being the best among candidate models. Models with a $\triangle A I C c<4$ were considered equally possible candidates and then their statistics averaged. The null model was included as a possible candidate but was never among the models with lowest AICc. Averaged model coefficients were obtained for models with a $\triangle A I C C<4$. Model inference and averaging were carried out with the R package 'MuMIn' 2 .

Principal Components analysis with Varimax Rotation: We realised a Principal Component Analysis ${ }^{3,4}$ with Varimax rotation using the R package 'Psych' ${ }^{5,6}$. Variables are automatically normalised. We set up three dimensions based on our previous researches on drawings. Varimax rotation is used to simplify the expression of a particular subspace in terms of just a few major items each. This means that the Varimax rotation turns the variables on the dimensions in order to maximise the explained variance. We examined loadings of each variable on each dimension. The loadings are interpreted as the coefficients of the linear combination of the initial variables from which the principal components are constructed. The loadings are equal to the coordinates of the variables divided by the square root of the eigenvalue associated with the component. 


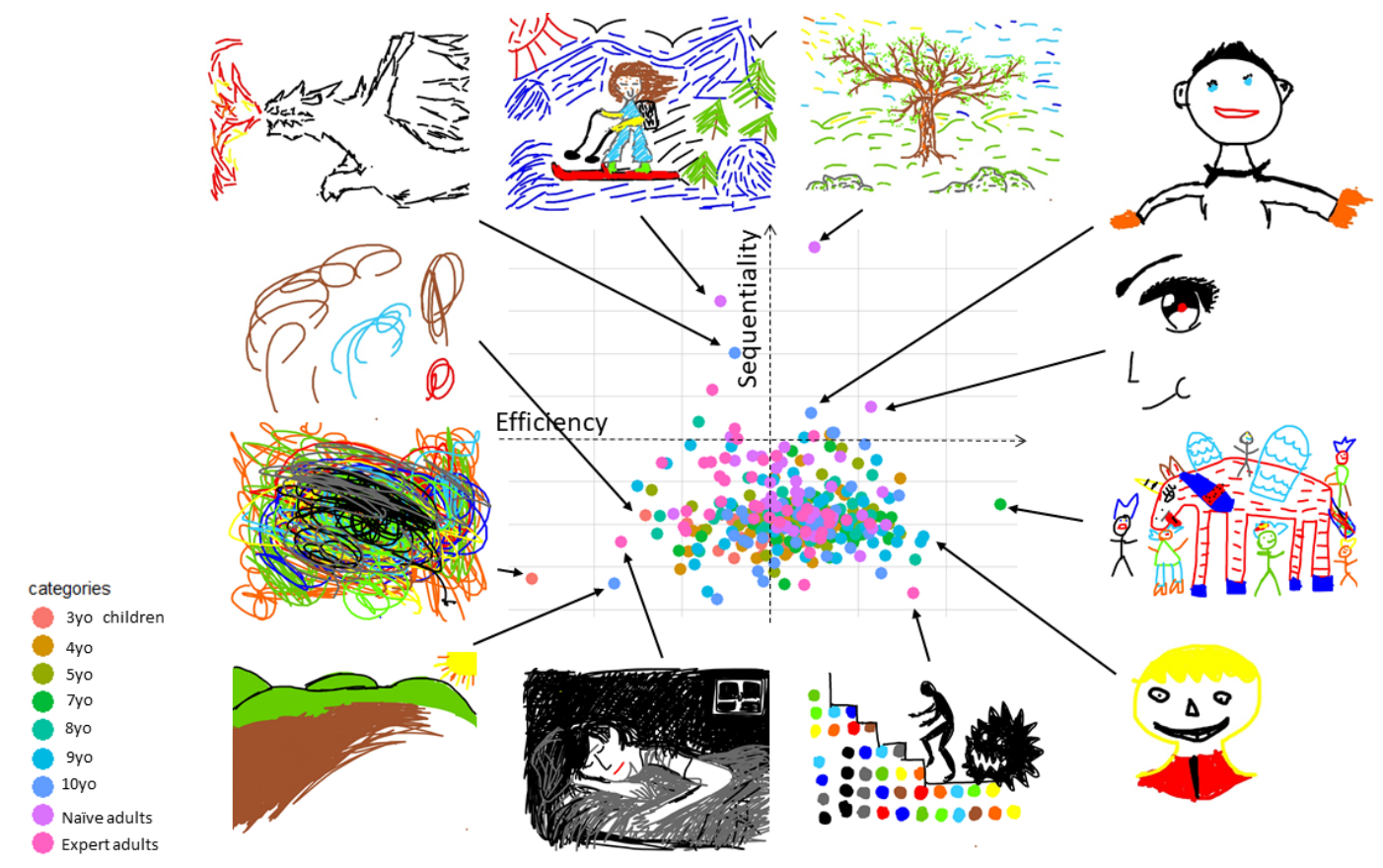

Figure S1: Instances of drawings for the graph Sequentiality in function of Efficiency.

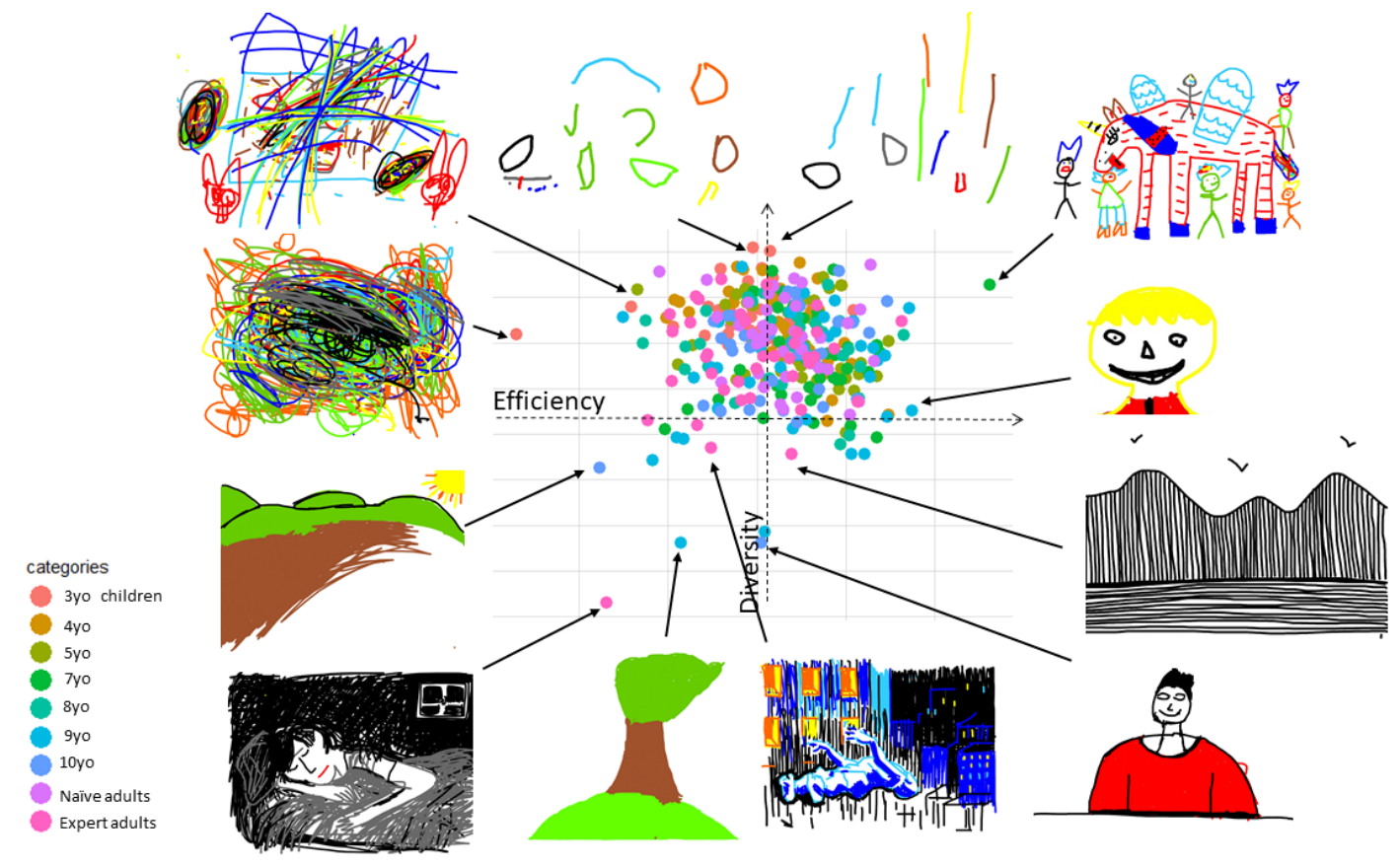

Figure S2: Instances of drawings for the graph Diversity in function of Efficiency. 


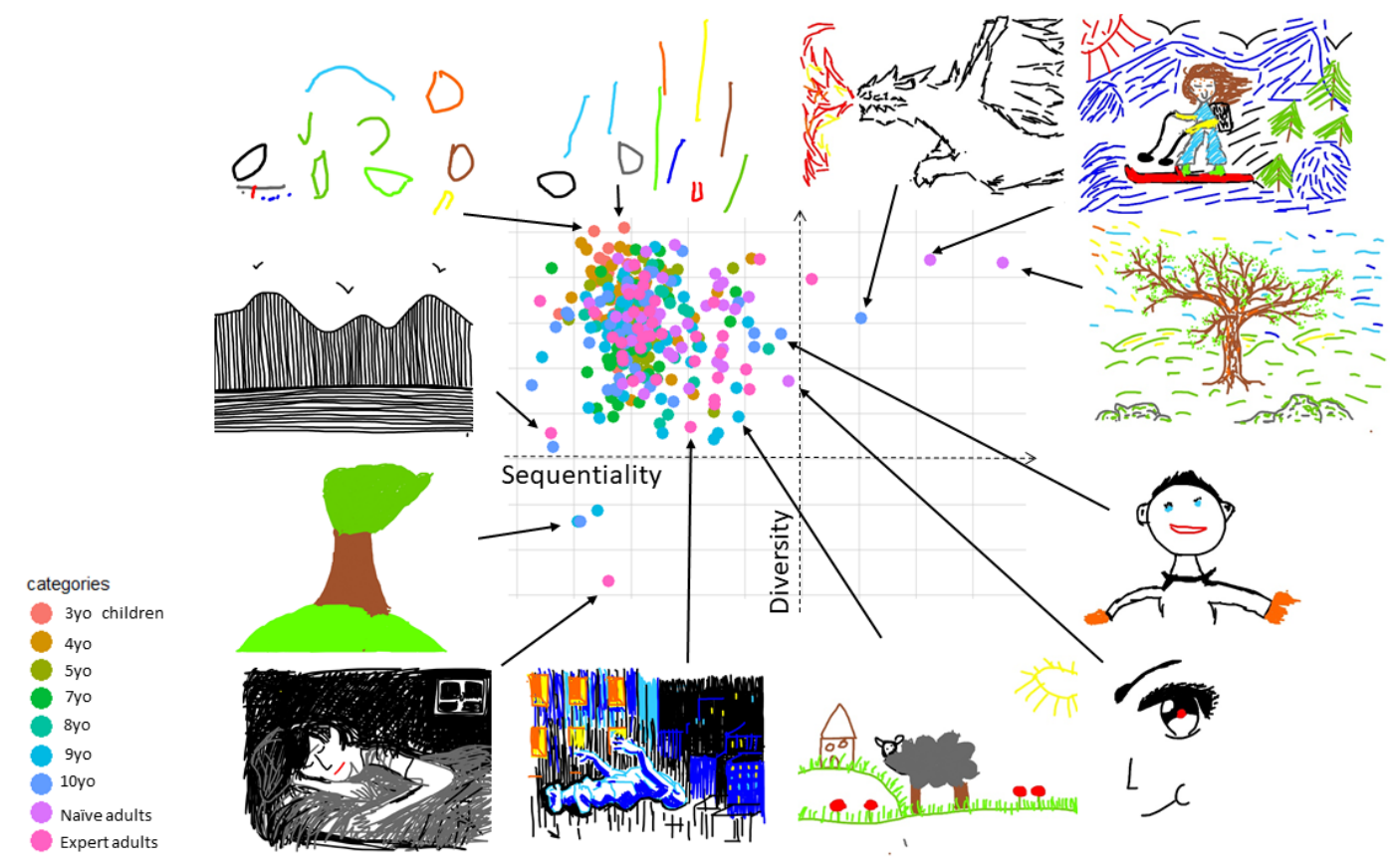

Figure S3: Instances of drawings for the graph Diversity in function of Sequentiality.

Table S1: Models retained in the selection model with the efficiency as dependent variables and the categories, the gender, the conditions and the gender-condition interaction and group-condition interaction as factors.

\begin{tabular}{|c|c|c|c|c|c|}
\hline Models & df & logLik & AICc & $\triangle \mathrm{AICC}$ & weight \\
\hline Categories & 10 & -100.46 & 221.59 & 0 & 0.38 \\
\hline $\begin{array}{r}\text { Categories + } \\
\text { conditions }\end{array}$ & 11 & -99.55 & 221.9 & 0.31 & 0.33 \\
\hline Categories + gender & 11 & -100.27 & 223.33 & 1.74 & 0.16 \\
\hline $\begin{array}{r}\text { Categories + } \\
\text { conditions }+ \text { gender }\end{array}$ & 12 & -99.35 & 223.65 & 2.06 & 0.14 \\
\hline
\end{tabular}

Table S2: Results of the averaged model with the efficiency as dependent variables following the model selection

\begin{tabular}{|l|r|r|r|r|r|} 
& \multicolumn{1}{c}{ Estimate } & \multicolumn{1}{c}{ Std. Error } & Adjusted SE & Z value & \multicolumn{1}{c}{ p-value } \\
\hline (Intercept) & 1.50521 & 0.057 & 0.0572 & 26.316 & $<0.0001$ \\
\hline categoriesb & -0.13929 & 0.07361 & 0.07388 & 1.885 & 0.05938 \\
\hline categoriesc & -0.21448 & 0.07361 & 0.07388 & 2.903 & 0.00369 \\
\hline categoriesd & -0.30567 & 0.07461 & 0.07488 & 4.082 & $<0.0001$ \\
\hline categoriese & -0.14455 & 0.07797 & 0.07825 & 1.847 & 0.0647 \\
\hline categoriesf & -0.1994 & 0.07446 & 0.07473 & 2.668 & 0.00762 \\
\hline categoriesg & -0.16295 & 0.07361 & 0.07388 & 2.206 & 0.02741 \\
\hline categoriesh & -0.17292 & 0.07461 & 0.07488 & 2.309 & 0.02093 \\
\hline categoriesi & 0.01804 & 0.07453 & 0.0748 & 0.241 & 0.80946 \\
\hline Freedrawing & 0.04718 & 0.03538 & 0.03551 & 1.329 & 0.18393 \\
\hline GenderMale & 0.02223 & 0.03604 & 0.03618 & 0.614 & 0.53891 \\
\hline
\end{tabular}


Table S3: Models retained in the selection model with the sequentiality as dependent variables and the categories, the gender, the conditions and the gender-condition interaction and group-condition interaction as factors.

\begin{tabular}{|c|c|c|c|c|c|}
\hline Models & df & logLik & AICc & $\triangle \mathrm{AICC}$ & weight \\
\hline Categories + gender & 11 & 51.39 & -79.99 & 0 & 0.41 \\
\hline Categories & 10 & 49.97 & -79.28 & 0.71 & 0.29 \\
\hline $\begin{array}{r}\text { Categories + } \\
\text { conditions + gender }\end{array}$ & 12 & 51.66 & -78.37 & 1.62 & 0.18 \\
\hline $\begin{array}{r}\text { Categories }+ \\
\text { conditions }\end{array}$ & 11 & 50.23 & -77.67 & 2.32 & 0.13 \\
\hline
\end{tabular}

Table S4: Results of the averaged model with the sequentiality as dependent variables following the model selection

\begin{tabular}{|l|r|r|r|r|r|} 
& \multicolumn{1}{c|}{ Estimate } & \multicolumn{1}{c}{ Std. Error } & \multicolumn{1}{c}{ Adjusted SE } & Z value & \multicolumn{1}{c}{ p-value } \\
\hline (Intercept) & 2.56429 & 0.03946 & 0.03958 & 64.782 & $<0.0001$ \\
\hline categoriesb & -0.06957 & 0.0478 & 0.04797 & 1.45 & 0.14696 \\
\hline categoriesc & -0.09149 & 0.0478 & 0.04797 & 1.907 & 0.05648 \\
\hline categoriesd & -0.05961 & 0.04851 & 0.04868 & 1.224 & 0.2208 \\
\hline categoriese & -0.13207 & 0.05046 & 0.05064 & 2.608 & 0.00911 \\
\hline categoriesf & -0.10457 & 0.04818 & 0.04835 & 2.163 & 0.03057 \\
\hline categoriesg & -0.09812 & 0.0478 & 0.04797 & 2.045 & 0.04082 \\
\hline categoriesh & -0.30497 & 0.04851 & 0.04868 & 6.264 & $<0.0001$ \\
\hline categoriesi & -0.19124 & 0.04832 & 0.0485 & 3.943 & $<0.0001$ \\
\hline Gendermale & -0.03864 & 0.02321 & 0.0233 & 1.659 & 0.09718 \\
\hline Freedrawing & 0.01629 & 0.02285 & 0.02293 & 0.71 & 0.47754 \\
\hline
\end{tabular}

Table S5: Models retained in the selection model with the diversity as dependent variables and the categories, the gender, the conditions and the gender-condition interaction and group-condition interaction as factors.

\begin{tabular}{|l|r|r|r|r|r|}
\hline Models & df & logLik & AICc & \multicolumn{1}{l}{$\Delta$ AICc } & weight \\
\hline Categories+conditions & 11 & -7.94 & 38.67 & 0 & 0.63 \\
\hline Categories+conditions+gender+conditions:gender & 13 & -6.32 & 39.73 & 1.07 & 0.37 \\
\hline
\end{tabular}


Table S6: Results of the averaged model with the diversity as dependent variables following the model selection

Estimate Std. Error Adjusted Z value p-value

\begin{tabular}{|l|r|r|r|r|r|}
\hline \multicolumn{7}{|c|}{ SE } \\
\hline (Intercept) & 2.10471 & 0.0447 & 0.04486 & 46.916 & $<0.0001$ \\
\hline categoriesb & -0.03548 & 0.0564 & 0.05661 & 0.627 & 0.53081 \\
\hline categoriesc & -0.14666 & 0.0564 & 0.05661 & 2.591 & 0.00958 \\
\hline categoriesd & -0.22639 & 0.05719 & 0.0574 & 3.944 & $<0.0001$ \\
\hline categoriese & -0.1591 & 0.05969 & 0.05991 & 2.656 & 0.00792 \\
\hline categoriesf & -0.31609 & 0.05701 & 0.05721 & 5.525 & $<0.0001$ \\
\hline categoriesg & -0.22846 & 0.0564 & 0.05661 & 4.036 & $<0.0001$ \\
\hline categoriesh & -0.11538 & 0.05719 & 0.0574 & 2.01 & 0.0444 \\
\hline categoriesi & -0.26246 & 0.05709 & 0.05729 & 4.581 & $<0.0001$ \\
\hline Freedrawing & 0.09323 & 0.03863 & 0.03873 & 2.407 & 0.01607 \\
\hline GenderMale & 0.01341 & 0.03866 & 0.0388 & 0.346 & 0.72953 \\
\hline Freedrawing:GenderMale & -0.08042 & 0.05428 & 0.05448 & 1.476 & 0.13995 \\
\hline
\end{tabular}

Table S7: P-values of age categories pairwise comparisons for the efficiency dimension

\begin{tabular}{|l|l|l|l|l|l|l|l|l|} 
& 3yo & 4yo & 5yo & 7yo & 8yo & 9yo & \multicolumn{1}{l}{ 10yo } & naive adults \\
\hline 4yo & 0.126 & - & - & - & - & - & - & - \\
\hline 5yo & $\mathbf{0 . 0 3 0}$ & 0.526 & - & - & - & - & - & - \\
\hline 7yo & $\mathbf{0 . 0 0 1}$ & 0.084 & 0.396 & - & - & - & - & - \\
\hline 8yo & 0.131 & 0.950 & 0.601 & 0.105 & - & - & - & - \\
\hline 9yo & $\mathbf{0 . 0 4 4}$ & 0.663 & 0.883 & 0.297 & 0.702 & - & - & - \\
\hline 10yo & 0.084 & 0.868 & 0.702 & 0.126 & 0.883 & 0.835 & - & - \\
\hline naive adults & 0.078 & 0.835 & 0.801 & 0.158 & 0.868 & 0.868 & 0.917 & - \\
\hline expert adults & 0.883 & 0.105 & $\mathbf{0 . 0 2 3}$ & $\mathbf{0 . 0 0 1}$ & 0.105 & $\mathbf{0 . 0 3 0}$ & 0.069 & 0.059 \\
\hline
\end{tabular}

Table S8: P-values of age categories pairwise comparisons for the sequentiality dimension

\begin{tabular}{|c|c|c|c|c|c|c|c|c|}
\hline & 3yo & 4yo & 5 yo & 7yo & 8 yo & 9yo & 10yo & naive adults \\
\hline 4yo & 0.322 & - & - & - & - & - & - & - \\
\hline 5 yo & 0.151 & 0.725 & - & - & - & - & - & - \\
\hline 7yo & 0.419 & 0.875 & 0.620 & - & - & - & - & - \\
\hline 8 yo & 0.038 & 0.351 & 0.576 & 0.273 & - & - & - & - \\
\hline 9 yo & 0.096 & 0.592 & 0.826 & 0.497 & 0.690 & - & - & - \\
\hline 10yo & 0.117 & 0.657 & 0.889 & 0.576 & 0.620 & 0.889 & - & - \\
\hline naive adults & $<0.0001$ & $<0.0001$ & $<0.0001$ & $<0.0001$ & 0.004 & $<0.0001$ & $<0.0001$ & - \\
\hline expert adults & 0.001 & 0.038 & 0.096 & 0.027 & 0.413 & 0.160 & 0.117 & 0.064 \\
\hline
\end{tabular}


Table S9: P-values of age categories pairwise comparisons for the diversity dimension

\begin{tabular}{|l|l|l|l|l|l|l|l|l|} 
& 3yo & 4yo & 5yo & 7yo & 8yo & 9yo & \multicolumn{1}{l}{ 10yo } & naive adults \\
\hline 4yo & 0.613 & - & - & - & - & - & - & - \\
\hline 5yo & $\mathbf{0 . 0 3 0}$ & 0.097 & - & - & - & - & - & - \\
\hline 7yo & $\mathbf{0 . 0 0 1}$ & $\mathbf{0 . 0 0 4}$ & 0.233 & - & - & - & - & - \\
\hline 8yo & $\mathbf{0 . 0 3 0}$ & 0.096 & 0.852 & 0.356 & - & - & - & - \\
\hline 9yo & $\mathbf{0 . 0 0 0}$ & $\mathbf{0 . 0 0 0}$ & $\mathbf{0 . 0 1 2}$ & 0.196 & $\mathbf{0 . 0 3 0}$ & - & - & - \\
\hline 10yo & $\mathbf{0 . 0 0 1}$ & $\mathbf{0 . 0 0 3}$ & 0.226 & 0.968 & 0.339 & 0.196 & - & - \\
\hline naive adults & $\mathbf{0 . 0 9 7}$ & 0.233 & 0.618 & 0.104 & 0.558 & $\mathbf{0 . 0 0 3}$ & 0.097 & - \\
\hline expert adults & $\mathbf{0 . 0 0 0}$ & $\mathbf{0 . 0 0 1}$ & 0.097 & 0.613 & 0.157 & 0.439 & 0.613 & \\
\hline
\end{tabular}

\section{References}

1. Burnham, K. P. \& Anderson, D. R. Multimodel inference understanding AIC and BIC in model selection. Sociol. Methods Res. 33, 261-304 (2004).

2. Bartoń, K. MuMIn: multi-model inference. R Package Version 1, (2013).

3. Budaev, S. V. Using principal components and factor analysis in animal behaviour research: caveats and guidelines. Ethology 116, 472-480 (2010).

4. Holland, S. M. Principal components analysis (PCA). Dep. Geol. Univ. Ga. Athens GA 30602-2501 (2008).

5. Revelle, W. An overview of the psych package. Dep. Psychol. Northwest. Univ. Accessed March 3, $1-25$ (2011).

6. Revelle, W. \& Revelle, M. W. Package 'psych'. Compr. R Arch. Netw. (2015). 